\title{
Paternal Obesity and Epigenetic Inheritance of Breast Cancer: The Role of Systemic Effects and Transmission to the Second Generation
}

\author{
Camile C. Fontelles
}

Georgetown University Medical Center

\section{Raquel Santana da Cruz}

Georgetown University Medical Center

Alexandra K. Gonsiewski

Georgetown University Medical Center

\section{Ersilia Barin}

Georgetown University Medical Center

\section{Volkan Tekmen}

Georgetown University Medical Center

\section{Lu Jin}

Georgetown University Medical Center

\section{Idalia Cruz}

Georgetown University Medical Center

\section{Olivier Loudig}

Center for Discovery and Innovation, Hackensack Meridian Health

\section{Anni Warri}

University of Turku

Sonia de Assis ( $\square$ deassiss@georgetown.edu )

Georgetown Lombardi Comprehensive Cancer Center https://orcid.org/0000-0001-5053-0614

\section{Research article}

Keywords: epigenetic inheritance, obesity, paternal programming, breast cancer, mammary gland, metabolic dysfunction, sperm non-coding RNAs

Posted Date: December 4th, 2020

DOl: https://doi.org/10.21203/rs.3.rs-118267/v1

License: (c) (1) This work is licensed under a Creative Commons Attribution 4.0 International License. Read Full License 


\section{Abstract}

Background: While genetics explains some familial breast cancer cases, we showed that environmentallyinduced epigenetic inheritance of breast cancer can also occur in rodent models. We previously reported that paternal consumption of a high-fat diet and ensuing obesity increased breast cancer susceptibility in the offspring (F1). Nevertheless, it is still unclear whether paternal-induced programming of breast cancer in daughters is associated with systemic alterations or mammary epithelium-specific factors. It also remains to be determined whether the ancestrally programmed breast cancer predisposition in F1 progeny can be transmitted to subsequent generations.

Methods: Male mice (F0) were fed either a control (CO) diet or an obesity-inducing diet (OID) for seven weeks and then mated with female mice (F0) reared on a CO diet. The resulting offspring (F1), also exclusively fed $\mathrm{CO}$ diet, were either used for mammary gland and tumor transplantation surgeries or to generate the F2 generation. To induce the mammary tumors, female mice were treated with 7,12 dimethylbenz[a]anthracene (DMBA). Total RNA extracted from F0 or F1 males sperm was used for small RNA-Seq analysis.

Results: Mammary glands from F1 CO female offspring exhibited enhanced development when transplanted into OID females [OID(CO-MG)], as shown by higher mammary gland area, epithelial branching and elongation, compared to $\mathrm{CO}$ females that received a $\mathrm{CO}$ mammary gland [CO(CO-MG)]. Similarly, mammary tumors from F1 CO female offspring transplanted into OID females [OID(CO.T)] displayed improved growth with a higher proliferation/apoptosis rate. We also found that granddaughters (F2) from the OID grand-paternal germline showed accelerated tumor growth compared to COxCO granddaughters (F2). Transmission of breast cancer predisposition to the F2 generation through OID male germline was associated with alterations in specific sperm tRNA fragments (tRF) in both F0 and F1 males.

Conclusions: Our findings indicate that systemic metabolic and mammary stromal alterations are the most significant contributors to paternal programming of mammary gland development and cancer predisposition in female offspring rather than mammary epithelium confined factors. Our data also show breast cancer predisposition in OID daughters can be transmitted to subsequent generations and could explain some familial cancers, if confirmed in humans.

\section{Introduction}

Genetic predisposition explains most but not all familial diseases, including breast cancer[1]. It is increasingly evident that epigenetic inheritance of disease can also occur and may explain some inherited conditions. There is strong indication that, at conception, parents pass more than genetic material to their offspring. They also transmit a molecular memory of past environmental exposures $[2,3]$ which can result in offspring's predisposition for certain chronic diseases [4]. 
Life-style and environmental insults have been shown to reprogram the sperm epigenome in humans and in animal models $[5,6]$. Recently published studies demonstrated that the small RNA load in paternal sperm can convey phenotypes to the progeny [3,7-9]. Some of those reports implicate t-RNA fragments (tRFs) - which are the most abundant small RNA sub-type in sperm-in the transmission environmentallyinduced information from fathers to offspring and show that they can recapitulate disease phenotypes [7-10].

Because mammary gland development starts during fetal development, multiple studies report that maternal exposure during gestation can epigenetically reprogram the daughters' mammary tissue and increase breast cancer development [11-14]. However, a role for paternal exposures in modulating breast cancer predisposition in offspring has emerged in recent years. We recently showed that paternal obesity, malnutrition and consumption of a high-fat diet all lead to increased breast cancer development in offspring [15-17], a phenotype associated with changes in normal mammary gland development. We also found that a recurrent phenotype accompanying offspring's cancer predisposition is metabolic dysfunction [16-18], raising the possibility that paternally-induced cancer development could be a function of both systemic effects as well as tissue specific changes.

Paternal effects on the F1 generation include alterations in the germline epigenome [19], suggesting that disease traits in offspring could be passed on to future generations. Indeed, it has been reported that paternally-induced phenotypes observed in the F1 can be transmitted to the F2 generation [19, 20]. It is still not clear, however, whether paternally-induced breast cancer predisposition observed in the offspring can be transmitted through successive generations without continuous exposure to the initial insult.

Here, we used a mouse model of paternal obesity and aimed to address the role of systemic metabolic alterations and the local mammary epithelial and/or stromal changes on breast cancer development in the F1 generation. We also investigated whether the breast cancer predisposition observed in daughters of obese fathers could be transmitted to granddaughters.

\section{Material And Methods}

\section{Dietary Exposures and Breeding}

The C57BL/6 mouse strain was used in all experiments. Male mice were fed AIN93G-based diets containing either 17.2\% (Control, CO, Envigo-Teklad \#TD160018) or 57.1\% (Lard-based, Obesity-InducingDiet, OID, Envigo-Teklad \#TD160019) energy from fat (Diet details in supplementary Table S1, see the section on supplementary data) starting after weaning (3 weeks of age). Males' body weight was recorded weekly (Fig. S1). At 10 weeks of age, OID-fed and CO-fed F0 male mice were mated with female mice reared solely on the $\mathrm{CO}$ diet to generate the F1 generation. Males were kept in female cages for 3 days. Female mice were kept on the $\mathrm{CO}$ diet during the breeding period, for the extent of pregnancy (21 days) and after giving birth. The birth weight and number of pups per litter were determined 2 days after

birth. To avoid litter-effect, pups were cross-fostered one day after dams gave birth. Pups from 2-3 dams 
were pooled and housed in a litter of 8-10 pups per nursing dam. All pups were weaned on postnatal day 21 and fed the $\mathrm{CO}$ diet throughout the experiment. Pups body weight was recorded weekly.

To obtain the F2 generation, F1 male offspring from OID fathers were mated with F1 females from either $\mathrm{CO}$ [OIDxCO] or OID [OIDxOID] groups. Similarly, F1 male offspring from CO fathers were mated with F1 females from either the $\mathrm{CO}$ [COxCO)] or OID [COxOID] groups. No sibling mating was carried out. F1 and F2 generation females from the $\mathrm{CO}$ or OID lineages were used to study body weight, metabolic function, mammary tumorigenesis and mammary transplantation, as described in the following sections. The experimental design is shown in Fig. $\mathbf{S 2}$.

F1 and F2 litters' gender distribution and number of offspring used in each experiment are shown in Table S2 and Table S3, respectively. All animal procedures were approved by the Georgetown University Animal Care and Use Committee, and the experiments were performed following the National Institutes of Health guidelines for the proper and humane use of animals in biomedical research. To increase rigor and reproducibility, animals were randomized to each experimental condition/ experiment and studies performed blindly where possible.

\section{Metabolic Function}

Insulin tolerance test (ITT) was performed after the mice fasted for $6 \mathrm{~h}$, according to the method described by Takada et al [21]. The insulin load ( $75 \mathrm{mU} / 100 \mathrm{~g}$ body weight) was injected as a bolus, and the blood glucose levels were determined at $0,3,6,9,12$, and 30 minutes after injection in female offspring. The area under the curve (AUC) was calculated according to the trapezoid rule. Differences in ITT were analyzed using two-way ANOVA (group, time), followed by post-hoc analyses.

\section{Mammary Transplantation}

Three-week old F1 female offspring of $\mathrm{CO}$ and OID males underwent a mammary gland transplantation surgery as previously described $[22,23]$. The experimental design is shown in Fig. S3. Females undergoing surgery were anesthetized using isoflurane flowing in oxygen and maintained with isoflurane flowing at $1-3 \%$. Before transplantation, the 4 th inguinal mammary gland of host females was cleared from their endogenous epithelium by removing the fat pad of the 4th gland up to its proximal lymph node. Special care was taken to cut off the connection between the 4th and 5th mammary glands to ensure complete clearing of the 4th mammary fat pad and to avoid later epithelial contamination from the 5th mammary gland. The excised fat pad containing the epithelial cells were stained with carmine aluminum solution to check cleared margins.

For transplantation, the donor fat pad containing the epithelial cells was excised and divided into small pieces $\left(1 \mathrm{~mm}^{3}\right)$ and placed into a tissue-culture plate containing DMEM/F12 to keep it moist. Mammary tissue fragments of the donor mouse, either CO or OID F1 female offspring, were then implanted into a pocket made in the cleared fat pad of the host (CO or OID). The skin incision was closed with surgical wound clips. The transplantations were performed from $\mathrm{CO}$ female offspring donors to both $\mathrm{CO}$ [CO(CO$M G)]$ and OID [OID(CO-MG)] female offspring hosts, as well as from OID female offspring donors to CO 
[CO(OID-MG)] female offspring hosts. Mammary glands transplants were collected approximately 10 weeks post-surgery and used for analysis of epithelial branching density, epithelial elongation and number of Terminal End Buds (TEBs) as described in the next sections.

\section{Transplanted mammary gland growth and development}

Transplanted mammary glands collected approximately 10 weeks post-surgery were stretched onto a slide, placed in a fixative solution and stained with a carmine aluminum solution (Sigma Chemical Co.) as previously described [24]. Whole mounts were examined under the microscope (AmScope) for ductal elongation and number of TEBs (undifferentiated structure considered to be the targets of malignant transformation), as previously described [24]. Whole-mount slides were also photographed (Olympus SZX12 250 Stereomicroscope), digitized and analyzed. Briefly, the portion surrounding the glandular epithelium was removed, color channels separated, and noise removed. The images were thresholded and skeletonized. Then, mammary epithelial area and branching (sum of intersections) were measured by Sholl analysis, a plugin ImageJ software (National Institute of Health, Bethesda, MD, USA) as previously described [25]. Once morphological analyses were completed, mammary whole mounts were removed from the slide, embedded in paraffin, sectioned $(5 \mu \mathrm{m})$ [26] and prepared for either hematoxylin and eosin (H\&E) or ki-67 staining as described below. Differences between groups were analyzed using one-way ANOVA, followed by post-hoc analyses.

\section{Mammary tumor induction}

Mammary tumors were induced in F1 an F2 female offspring by administration of medroxyprogesterone acetate (MPA; $15 \mathrm{mg} / 100 \mu \mathrm{l}$, subcutaneously) to 6 weeks of age female offspring, followed by three weekly doses of $1 \mathrm{mg}$ 7,12-dimethylbenz[a]anthracene (DMBA; Sigma, St. Louis, MO) dissolved in peanut oil by oral gavage[27]. Tumors were detected by palpation once per week, starting at week 2 after the last dose of DMBA. Tumor growth was measured using a caliper, and the width and height of each tumor were recorded.

In the F1 generation, mammary tumors were harvested when reaching approximately $40 \mathrm{~mm}^{2}$ in volume and used for mammary tumor transplantation surgery, as described in the next section. In the F2 generation, tumor development was monitored for a total of 20 weeks post-DMBA administrations. Animals in which tumor burden reached approximated $10 \%$ of total body weight were euthanized before the end of the monitoring period, as required by our institution. Tumor growth was analyzed using twoway ANOVA (group and time), followed by post-hoc analyses. Kaplan-Meier survival curves were used to compare differences in tumor incidence, followed by the log-rank test. Differences in tumor latency and mortality were analyzed using two-way ANOVA.

\section{Mammary tumor transplantation}

$\mathrm{CO}$ and OID F1 female offspring underwent a mammary tumor transplantation surgery at approximately 11 weeks of age. Females undergoing surgery were anesthetized using isoflurane flowing in oxygen and maintained with isoflurane flowing at $1-3 \%$. Briefly, carcinogen-induced mammary tumor fragments (1 
$\mathrm{mm}^{3}$ ) of a donor mouse, either CO or OID offspring, were implanted into a pocket made in the mammary fat pad of the host (CO or OID). The experimental design is shown in Figure S3. Mammary tumors grown from the transplants were collected approximately 6-8 weeks post-surgery. Differences between groups were analyzed using one-way ANOVA, followed by post-hoc analyses.

\section{Analysis of cell proliferation}

Cell proliferation (Ki-67) was evaluated by immunohistochemistry in F1 mammary gland and mammary tumors transplants as well as F2 mammary tumors. Briefly, tissues were fixed in $10 \%$ buffered formalin, embedded in paraffin, and sectioned $(5 \mu \mathrm{m})$. Sections were deparaffinized with xylene and rehydrated through a graded alcohol series. Antigen retrieval was performed by immersing the tissue sections at $98{ }^{\circ} \mathrm{C}$ for 40 minutes in $1 \mathrm{X}$ Diva Decloaker (Biocare). Tissue sections were treated with $3 \%$ hydrogen peroxide and $10 \%$ normal goat serum for 10 minutes and were incubated with the primary antibody, overnight at $4{ }^{\circ} \mathrm{C}$. After several washes, sections were treated to the appropriate HRP labeled polymer for 30 min and DAB chromagen (Dako) for 5 minutes. Slides were counterstained with Hematoxylin (Fisher, Harris Modified Hematoxylin), blued in $1 \%$ ammonium hydroxide, dehydrated, and mounted with Acrymount. The sections were photographed using an Olympus IX-71 Inverted Epifluorescence microscope at 40x magnification. Proliferation index (Ki-67 staining) was determined by immunoRatio, a plugin Image $\mathrm{J}$ software (National Institute of Health, Bethesda, MD, USA), to quantify hematoxylin and DAB-stained cells. Differences between groups were analyzed using one-way ANOVA, followed by posthoc analyses.

\section{Analysis of cell apoptosis}

Cell apoptosis analysis was performed in F1 transplanted mammary glands and tumors and F2 mammary tumors by morphological detection. Tissues were fixed in neutral buffered $10 \%$ formalin, embedded in paraffin, sectioned $(5 \mu \mathrm{m})$ and stained with hematoxylin and eosin (H\&E). Cells presenting loss of adhesion between adjacent cells, cytoplasmic condensation and formation of apoptotic bodies were considered apoptotic as described before[28]. Sections were photographed using an Olympus IX-71 Epifluorescence microscope at 40x magnification. Twenty areas were photographed randomly, and the number of apoptotic bodies counted. Images were evaluated with ImageJ software (NIH, USA). Differences between groups were analyzed using one-way ANOVA, followed by post-hoc analyses.

\section{Mature spermatozoa collection and purification}

$\mathrm{CO}$ and OID-fed males (F0) and their male offspring (F1) were euthanized and their caudal epididymis dissected for sperm collection. The epididymis was collected, punctured, and transferred to tissue culture dish containing M2 media (M2 Medium-with HEPES, without penicillin and streptomycin, liquid, sterilefiltered, suitable for mouse embryo, SIGMA, product \#M7167) where it was incubated for 1 hour at $37^{\circ} \mathrm{C}$.

Sperm samples were isolated and purified from somatic cells. Briefly, the samples were washed with PBS, and then incubated with SCLB (somatic cell lysis buffer, $0.1 \%$ SDS, $0.5 \%$ TX-100 in Diethylpyrocarbonate water) for 1 hour. SCLB was rinsed off with 2 washes of PBS and the somatic cell-free purified spermatozoa sample pelleted and used for RNA extraction. 


\section{Small RNA-Seq and Gene Ontology (GO) analyses}

Total RNA was isolated from sperm using Qiagen's miRNeasy extraction kit, according to the manufacturer's instructions. One hundred ng of column-purified sperm RNA was used to prepare individually barcoded small-RNA libraries. Samples were barcoded, pooled, precipitated and separated on a $15 \%$ polyacrylamide gel (PAGE). The gel was stained with SYBR® gold dye and the small non-coding RNA segment corresponding to transfer RNA fragments or tRFs (30-45 nucleotides) excised and purified using a cDNA library preparation method described previously [29]. This library preparation method was demonstrated to be highly reproducible using total RNA with RNA Integrity Numbers as low as 2.0[29]. Indexed, single-ended small-RNA sequencing libraries were prepared. For each individual barcoded library, at least 10 million reads (raw data) were generated using an Illumina Hi-Seq 2500. The raw reads were subjected to 3 ' adapter trimming and low quality filtering using Trimmomatic program [30]. The high quality clean reads (Data quality control is shown in Fig. S4) were aligned to the mouse genome. tRFs tags were mapped to the mouse genome (GRCm38/mm10 reference genome) in order to analyze their genomic distribution and expression in the different sperm RNA samples. Small RNA tags were annotated and aligned to known t-RNA sequences using Ref-seq, GenBank and Rfam database using blastn with standard parameters. To analyze the differential expression of tRFs between $\mathrm{CO}$ and OID groups, tRFs were normalized to TPM (Transcripts Per Kilobase Million). tRFs with a P value less than 0.05 were considered significant, with an appropriate correction for multiple testing [31]. Target genes for the 5 overlapping tRFs in OID F0 and F1 males were predicted using TargetScan Mouse custom seedmatch and modified miRanda algorithm (energy $<=-20$ and score $>=150$ ). The common predicted genes were then uploaded to PANTHER 15.0 for GO term and pathway analysis, final lists were filtered by FDR $<0.25$.

\section{Results}

\section{Offspring of OID fathers have impaired metabolic function and altered mammary gland development}

We previously reported that paternal consumption of obesity-inducing diets (OID) at the pre-conception window increased female offspring's susceptibility to breast cancer $[15,16]$. In those studies, we also described mammary gland morphological changes as well as metabolic dysfunction-a phenotype also reported by others-in offspring of obese fathers $[16,18,19,32]$. Our present results corroborate our previous findings as OID offspring (F1) displayed impaired metabolic function with both F1 males and females showing significantly higher glucose levels in an insulin tolerance test (ITT) compared to CO offspring ( $P=0.002, P=0.011$, Fig. 1a-f). In addition, mammary glands of OID daughters also showed increased number of terminal end buds (TEB), higher epithelial branching and elongation, although only the last parameter reached statistical significance compared to CO (Table S4). Those phenotypes were not associated with body weight gain (Fig. S5) as OID offspring weights either did not differ from or were lower than $\mathrm{CO}$. 


\section{Systemic effects play a larger role in normal mammary tissue and mammary tumor growth in offspring of OID fathers}

Next, we examined the contributions of systemic alterations and mammary tissue specific factors (stroma vs. epithelium) to the increased breast cancer development in offspring of obese fathers. In the first experiment, female offspring of either $\mathrm{CO}$ or OID-fed males underwent a mammary gland transplantation surgery. CO mammary glands transplanted into OID females [OID(CO.MG)] exhibited accelerated development (Fig. 2a-e) as shown by higher mammary gland area $(p=0.032$, Fig. 2b), higher mammary branching and higher epithelial elongation $(p=0.014 ; p=0.008$, respectively, Fig. $2 c-d)$, but not higher number of TEBs (Fig. 2e), compared to $\mathrm{CO}$ females that received a CO mammary gland $[\mathrm{CO}(\mathrm{CO} . \mathrm{MG})]$. This phenotype was associated with a higher proliferation index and lower apoptotic rates compared to compared to $[\mathrm{CO}(\mathrm{CO} . \mathrm{MG})]$ ) and $[\mathrm{CO}(\mathrm{OID} . \mathrm{MG})](\mathrm{P}=0.021$ and $\mathrm{P}=0.026$, respectively; Fig. $2 \mathrm{f}$ j). While OID mammary glands transplanted into CO females [CO(OID.MG)] showed slightly higher mammary gland area, mammary branching and epithelial elongation and number of TEBS (Fig. 2b-e) compared to $[\mathrm{CO}(\mathrm{CO} . \mathrm{MG})]$, results did not reach statistical significance.

Given that both the mammary microenvironment and systemic response could play a role in tumor progression, we also asked whether the metabolic-induced mammary stroma milieu could affect the growth potential of tumors. Thus, in our second experiment, a DMBA-induced mammary tumor of F1 female offspring from $\mathrm{CO}$ (donor) was transplanted into the fat pad of a CO or OID female offspring (host) and vice versa. Tumor growth was followed for 6-8 weeks post-surgery. While not statistically significant (but in line with what we observed for mammary gland transplants), CO tumors transplanted into OID females [OID(CO.T)] displayed improved growth (Fig. 3a) and shorter latency (Fig. 3b) compared to $\mathrm{CO}$ or OID tumors transplanted in CO females [CO(CO.T) and CO (OID.T)]. [OID(CO.T)] tumor also showed significantly increased cell proliferation to apoptosis ratio, compared to both [CO(CO.T)] and [CO (OID.T)] ( $p=0.043, P=0.032$, respectively; Fig. 3c-g).

\section{Consumption of OID alters the tRF content in sperm of fathers (F0) and their sons (F1)}

Recent studies have suggested that sperm non-coding RNAs play a role in transmitting environmentallyinduced information from fathers to offspring. Transfer RNA fragments or tRFs make up the majority of small RNAs in mature sperm and can recapitulate the effects of paternal obesity in offspring [3]. As reported before, GlyGCC and GlutCTC were the most abundant tRFs in sperm of both fathers (F0) and their male offspring (F1), representing about $70 \%$ of all tRFs (Fig. 4a-b) $[8,19]$. We also found that consumption of OID altered specific tRFs in both father (Fig. 4c) and sons (Fig. 4d), with five tRFs overlapping between the two generations (Fig. 4e, Fig. S6): Levels of ValTAC and SerCGA were increased while those of ArgCCG, ArgTCG and SeCTCA were decreased in sperm of OID F0 and F1 males compared 
to CO. Putative targets of these five tRFs were significantly enriched for molecular functions related to DNA binding, transcription factor activity, transcriptional regulation, and transmembrane transporters among others (Fig. 4f).

\section{Breast cancer predisposition in OID daughters is transmitted to a second generation}

Given the tRF alterations observed in the F1 OID offspring germline, we then asked whether breast cancer predisposition in OID daughters could be inherited by a second generation of females. To this question, we produced the F2 generation by mating F1 male offspring from OID fathers with F1 females from either $\mathrm{CO}$ [OIDxCO] or OID [OIDxOID] groups. Similarly, F1 male offspring from $\mathrm{CO}$ fathers were mated with F1 females from either the $\mathrm{CO}$ [COxCO)] or OID [COxOID] groups (Fig.S2). Indeed, we found that the female F2 generation derived from either the F1 OID male and female lineage (OIDxCO and COxOID, respectively) or both (OIDxOID) developed carcinogen-induced mammary tumors that grew significantly faster, compared to COxCO group ( $p<0.001$, Fig. 5a). The incidence of mammary tumors at the end of the monitoring period was also significantly higher in F2 OIDxOID females compared to the COxCO group ( $p$ $=0.037$; Fig. $5 b$ ), suggesting a synergistic effect of both the male and female OID germlines. Tumor latency and tumor mortality rates in the OIDxCO group were slightly shorter than in all other groups, however results did not reach statistical significance (Fig. 5c-d).

Given the increased tumor growth in the F2 generation derived from the OID lineage (Fig. 5a), we next investigated cell proliferation and apoptosis rates in F2 mammary tumors (Fig. 5e-i.) We observed a nonsignificant increase in cell proliferation and a decrease in apoptotic indices in $\mathrm{F} 2$ females tumors derived from the OID lineage compared to COXCO F2 offspring (Fig. $5 \mathrm{~g}-\mathrm{h}$ ). Overall, tumors from OID groups (OIDxCO and COxOID, OIDxOID) also showed significantly higher proliferation/apoptosis ratio $(\mathrm{P}=0.032$; Fig. 5i.). Between-group comparisons showed that the OIDxOID group had significantly lower tumor apoptosis levels and higher tumor proliferation/apoptosis ratio compared to the COxCO group $(P=0.013$ and $\mathrm{P}=0.004$, respectively; Fig. 5h-i).

While all F2 females derived from the OID grand-paternal lineage (COxOID, OIDxCO, OIDxOID) showed higher mammary tumor growth with significantly larger tumors (Fig. $5 \mathrm{a}$ ) when compared to $\mathrm{COxCO}$, only OIDxOID females developed impaired insulin sensitivity as shown by higher glucose levels in ITT and AUC values $(P=0.007, P=0.017$, Fig. $5 j-k)$. However, OIDxCO females were significantly heavier overtime compared to all other groups (COxCO, COxOID, OIDxOID, $\mathrm{P}=0.0004$, Fig. S7).

\section{Discussion}

We previously reported that paternal obesity increases tumorigenesis in offspring, including breast cancer $[15,16,18]$. Our findings in this follow-up study suggest that metabolic disturbances in the F1 generation play a key role in contributing to the enhanced breast cancer growth observed in offspring of obese fathers in a mouse model. We also report that the paternal obesity leads to higher cancer development in two successive generations. Transmission of the increased breast cancer phenotype into the F2 
generation was associated with epigenetic changes in the germline, namely alterations in the abundance of specific tRFs present in OID F1 male sperm.

The first aim of our study was to dissect the distinct contributions of systemic effects and mammary tissue-confined factors to increased breast cancer development in daughters of obese fathers, as we had observed both metabolic dysfunction and mammary gland abnormalities in previous studies[15, 16]. In this study, we confirmed previous findings and show that females offspring of obese fathers showed higher circulating levels of glucose during an insulin tolerance test. While the data suggest that OID daughters are insulin intolerant, it is also possible that OID offspring have a higher capacity to store glucose (i.e. higher glycogen levels in the liver). More importantly, our results suggest those systemic metabolic effects, likely acting though the mammary stroma, in OID daughters play a larger role compared to the mammary epithelium. Further, tumors from $\mathrm{CO}$ offspring transplanted into OID daughters acquired a growth advantage compared to those transplanted in controls, suggesting that the stroma in OID females allows for better implantation and tumor growth. It is still possible that mammary epithelium confined factors play a role in the increased tumor development in OID offspring, however, they play a reduced role compared to systemic and mammary stromal effects according to our data. While it has been traditionally thought that the epithelium is the compartment with the dominant contribution regarding breast cancer initiation and growth and mammary tissue regeneration, some studies have highlighted the importance the stroma microenvironment, particularly adipocytes, on normal mammary development and malignant transformation of the mammary epithelium[33-36]. Our analyses are in agreement with those findings and suggest that the stroma plays an important enabling role for tumor growth.

It is also well established in epidemiologic studies that metabolic conditions such as obesity, metabolic syndrome and diabetes are important risk factors for breast cancer and other malignancies [37-40] and data from animal models offer support to those findings [41, 42]. In line with that, our study suggests that a milieu of metabolic dysfunction and altered stromal microenvironment creates conditions for increased proliferation and survival of both normal and tumorigenic mammary cells as demonstrated by our transplantation studies.

While we have not directly investigated the molecular mechanisms behind the findings reported here, it is known that metabolic dysfunction contributes to cancer development via extrinsic and tumor-intrinsic factors [43]. Metabolic-induced alterations in growth factors signaling, inflammation and the associated microenvironment, as well as changes in tumor metabolism itself are all major contributors to cell proliferation and cancer development [43]. Not surprisingly, our previously reported results show that paternal obesity or malnutrition alters the molecular make-up of tumors which show increased growth factor and energy sensing signaling and altered amino-acid metabolism[15-18].

We also examined whether the offspring's breast cancer predisposition programmed by paternal obesity could be inherited by a second unexposed generation. We found that the risk of breast cancer is passed down to the OID grandchildren equally via the F1 male and female germlines. Our data also suggest that 
there is a synergistic effect when both F1 parents had an obese father, with their descendants showing not only accelerated tumor growth but also higher tumor incidence. As with the F1 generation, F2 females from the OID lineage showed signs of metabolic dysfunction which depended whether they originated from the male or female lineage or both.

Our study offers some insights into the potential mechanism of transmission of breast cancer risk from one generation to another. Given the increased mammary tumorigenesis in the granddaughters of OID males in the absence of any further exposure, transmission of this phenotype conceivably occurs via F1 germ cells, which give rise to the F2 generation. In support of that, we found that F1 male germline showed alteration in tRFs, a class of small non-coding RNAs abundant in sperm, recently shown to transmit environmentally-induced information from one generation to another [7, 8]. While details on the functional role of tRFs in embryonic development are still under investigation, these small RNAs have been implicated in the regulation of translation, stress granule formation, viral replication and retrotransposons[44, 45]. Unfortunately, the inherent technical challenge of collecting enough eggs for molecular analysis precluded us from evaluating the F1 female germline. However, given that both the F1 male and female OID germline were able to transmit the increased predisposition to breast cancer phenotype to a second generation it is likely that we would have observed changes in the female germline as well. Nevertheless, we cannot rule out that some of the effects observed in F2 generation are due to maternal metabolic dysfunction in pregnancies of F1 OID females.

Interestingly, we found overlap in tRFs altered in sperm of F1 and F0 males. This suggests either that the F1 male germline is programmed by paternal obesity or that sperm non-coding RNAs are re-set in the F1 generation. Although, no changes in body weight were detected in F1 OID males, they did show metabolic dysfunction (impaired insulin sensitivity) later in life. However, others have shown that changes in the germline of male offspring of obese fathers occur in the absence of overt metabolic dysfunction[19], suggesting that F2 generation phenotypes represent true epigenetic inheritance.

The mechanisms for how germline epigenetic programming lead to phenotypes in offspring are still being investigated. The functions of tRFs are not yet fully understood due to their recent discovery. However, these small RNAs play roles in different biological processes including regulation of gene expression at the transcriptional, post-transcriptional, and translational level [6]. tRFs also play a role in RNA metabolism, global and transcript-specific translation inhibition ribosome biogenesis targeted cleavage of 3' UTRs, regulation of apoptosis, and regulation of retroviral elements[6]. Given the short halflife of sperm small non-coding RNAs such as tRFs, is likely that they act early in embryonic development, setting a cascade of molecular events which biases cellular programming during subsequent divisions and culminate in disease phenotypes $[3,6]$. Our gene ontology analysis of targets of the five overlapping tRFs in OID F0 and F1 OID males' sperm showed an enrichment for functions related to DNA binding, transcription factor activity, transcriptional regulation, and transmembrane transporters. It is possible that an imbalance in the amount of those specific tRFs in sperm can disrupt embryonic development postfertilization, programming the organism to be more to be more amenable and tolerant to cellular growth 
which would translate in increased cancer development. The exact mechanisms, however, need to be further investigated in follow-up studies.

\section{Conclusions}

In conclusion, the findings described here builds on our previous works and suggest that paternallyinduced cancer development is largely due to systemic alterations in offspring and that the offspring's breast cancer predisposition, as evaluated in this study, can be transmitted to a subsequent generation. While our study was conducted in an animal model, it could have important implications for human health. It is well known that family history is a strong predictor of cancer risk [46], yet not all familial cancers can be explained by genetic mutations[1,47]. Though it is estimated the up to $30 \%$ of breast cancers cluster in families, only about one third of those are due to mutations in high penetrance genes such as $B R C A 1$ and $B R C A 2$, leaving a sizable portion of familial breast cancers without a biological explanation [48]. Our study suggests that ancestral history of obesity from the paternal lineage could account for some familial cancers and that some organisms may be predisposed to the tolerance of cancer cells or may provide adequate conditions for their growth and development. This notion is supported by our prior findings showing that maternal exposure to an endocrine disruptor or dietary fat can also lead to multigenerational risk of breast cancer through both the male and female germlines in rats[12]. Given that the current study was performed in mice, our findings have now been confirmed in two different animal species.

It is also important to note that conditions such as obesity and malnutrition often occur in minorities and disadvantaged populations [49]. Our findings would suggest that social determinants of cancer predisposition and outcomes may be imprinted even before birth and are epigenetically mediated. However, it remains to be determined whether the biological insights uncovered by our study can account for some of the familial breast cancer predisposition or cancer disparities in humans.

\section{Abbreviations}

AUC - Area under the curve; BRCA - Breast Cancer gene; CO - control diet; DMBA - 7,12 dimethylbenz[a]anthracene; F0 - Parental generation; F1 - First filial generation; F2 -Second filial generation; GO - Gene Ontology; H\&E - Hematoxylin and eosin; ITT - Insulin tolerance test; MPA Medroxyprogesterone acetate; OID - obesity-inducing diet; SCLB - Somatic cell lysis buffer; TEBs - terminal end buds; tRFs - tRNA-derived fragments; [OID(CO-MG)]- mammary gland from F1 CO female offspring transplanted into OID female offspring, [CO(OID-MG)]-mammary gland from F1 OID female offspring transplanted into $\mathrm{CO}$ female offspring; [OID(CO-MG)]- mammary tumor from $\mathrm{F} 1 \mathrm{CO}$ female offspring transplanted into OID female offspring; [CO(OID.T)]-mammary tumor from F1 OID female offspring transplanted into CO female offspring.

\section{Declarations}


Ethics approval and consent to participate:

All animal procedures were approved by the Georgetown University Animal Care and Use Committee (protocol \# 2016-1172), and the experiments were performed following the National Institutes of Health guidelines for the proper and humane use of animals in biomedical research.

\section{Consent for publication:}

All authors approved of the manuscript and consented to its publication.

\section{Availability of data and materials:}

The small RNA-seq data has been deposited in GEO (Gene Expression Omnibus) database with accession code GSE161831.

\section{Competing interests:}

The authors declare no competing financial interests.

\section{Funding:}

This study was supported by The American Cancer Society (RSG-16-203-01-NEC, Research Scholar Grant to S. de Assis), and the National Institutes of Health (1P30-CA51008; Lombardi Comprehensive Cancer Center Support Grant to Louis Weiner and CCSG pilot fund to S. de Assis).

\section{Author contributions:}

C.C.F, A.W. and S.D.A. conceived the study. S.DA. oversaw the research and wrote the manuscript with the help of C.C.F, R.S.C., A.W. and O.L.; C.C.F performed most of the experiments with the help of A.W., R.S.C., A.K., E.B. M.I.C. and V.T.; C.C.F. and R.S.C. analyzed the data; O.L. performed the sperm small RNA-seq profiling and L.J. performed the RNA-seq data and GO analysis.

\section{Acknowledgments:}

We thank the following Lombardi Cancer Center Shared Resources (SR) for their assistance: Animal Model SR, Histopathology \& Tissue SR, Microscopy and Imaging SR.

\section{References}

1. Aalen 00 , Valberg $M$, Grotmol T, Tretli S: Understanding variation in disease risk: the elusive concept of frailty. Int J Epidemio/2015, 44(4):1408-1421.

2. OJ R: Daddy issues: paternal effects on phenotype. Cell 2012, 151(4):702-708.

3. Chen Q YW, Duan W: Epigenetic inheritance of acquired traits through sperm RNAs and sperm RNA modifications. Nature Reviews Genetics 2016, 17. 
4. MK JRS: Environmental epigenomics and disease susceptibility. Nature Review Genetics 2007, 8(4):253-262.

5. Donkin I VS, Ingerselv LR, Qian K, Mechta M, Nordkap L, Mortensen B, Appel EV, Jørgensen N, Kristiansen VB, Hansen T, Workman CT, Zierath JR, Barrès R: Obesity and Bariatric Surgery Drive Epigenetic Variation of Spermatazoa in Humans. Cell Metabolism 2016, 23(2):369-378.

6. Sharma U: Paternal Contributions to Offspring Health: Role of Sperm Small RNAs in Intergenerational Transmission of Epigenetic Information. Front Cell Dev Biol 2019, 7:215.

7. Chen Q YM, Cao Z, Li X, Zhang Y, Shi J, Feng GH, Peng H, Zhang X, Zhang Y, Qian J, Duan E, Zhai Q, Zhou Q: Sperm tsRNAs contribute to intergenerational inheritance of an acquired metabolic disorder. Science 2016, 351(6271):397-400.

8. Sharma U CC, Shea JM, Boskovic A, Derr AG, Bing XY, Belleannee C, Kucukural A, Serra RW, Sun F, Song L, Caronne BR, Ricci EP, Li XZ, Fauquier L, Moore MJ, Sullivan R, Mello CC, Garber M, Rando OJ: Biogenesis and function of tRNA fragments during sperm maturation and fertilization in mammals. Science 2016, 351(6271):391-396.

9. Gapp K JA, Sarkies P, Bohacek J, Pelzcar P, Prados J, Farinelli L, Miska E, Mansuy IM: Implication of sperm RNAs in transgenerational inheritence of the effects of early trauma in mice. Nature Neuroscience 2014, 17(5):667-669.

10. Rodgers $A B M C$, Leu NA, Bale TL: Transgenerational epigenetic programming via sperm microRNA recapitulates effects of paternal stress. Proceedings of the National Academy of Sciences of the United States of America 2015, 112(44):13699-13704.

11. de Assis S KG, Hilakivi-Clarke L: High birth weight increases mammary tumorigenesis in rats. International Journal of Cancer 2006, 119(7):1537-1546.

12. de Assis S WA, Cruz MI, Laja O, Tian Y, Zhang B, Wang Y, Huang T, Hilakivi-Clarke L: High-fat or ethinyl-oestradiol intake during pregnancy increases mammary cancer risk in several generations of offspring. Nature Communications 2012, 3.

13. Cohn BA LMM, Krigbaum NY, Yeh G, Park JS, Zimmermann L, Cirillo PM: DDT Exposure in Utero and Breast Cancer. Journal of Clinical Endocrinology and Metabolism 2015, 100(8):2865-2872.

14. de Oliveira Andrade F, Fontelles CC, Rosim MP, de Oliveira TF, de Melo Loureiro AP, Mancini-Filho J, Rogero MM, Moreno FS, de Assis S, Barbisan LF et al: Exposure to lard-based high-fat diet during fetal and lactation periods modifies breast cancer susceptibility in adulthood in rats. $J$ Nutr Biochem 2014, 25(6):613-622.

15. Fontelles CC, Carney E, Clarke J, Nguyen NM, Yin C, Jin L, Cruz MI, Ong TP, Hilakivi-Clarke L, de Assis $\mathrm{S}$ : Paternal overweight is associated with increased breast cancer risk in daughters in a mouse model. Sci Rep 2016, 6:28602.

16. Fontelles CC, Guido LN, Rosim MP, Andrade Fde O, Jin L, Inchauspe J, Pires VC, de Castro IA, HilakiviClarke L, de Assis $S$ et al: Paternal programming of breast cancer risk in daughters in a rat model: opposing effects of animal- and plant-based high-fat diets. Breast Cancer Res 2016, 18(1):71. 
17. da Cruz RS, Carney EJ, Clarke J, Cao H, Cruz MI, Benitez C, Jin L, Fu Y, Cheng Z, Wang Y et al: Paternal malnutrition programs breast cancer risk and tumor metabolism in offspring. Breast Cancer Res 2018, 20(1):99.

18. da Cruz RS, Clarke J, Curi AC, Al-Yawar A, Jin L, Baird A, Cruz I, Kallakury BV, de Assis S: Parental obesity programs pancreatic cancer development in offspring. Endocr Relat Cancer 2019.

19. Cropley JE, Eaton SA, Aiken A, Young PE, Giannoulatou E, Ho JWK, Buckland ME, Keam SP, Hutvagner G, Humphreys DT et al: Male-lineage transmission of an acquired metabolic phenotype induced by grand-paternal obesity. Mol Metab 2016, 5(8):699-708.

20. Wei Y, Yang CR, Wei YP, Zhao ZA, Hou Y, Schatten H, Sun QY: Paternally induced transgenerational inheritance of susceptibility to diabetes in mammals. Proc Natl Acad Sci U S A 2014, 111(5):18731878.

21. Takada J, Fonseca-Alaniz MH, de Campos TB, Andreotti S, Campana AB, Okamoto M, Borges-Silva C, Machado UF, Lima FB: Metabolic recovery of adipose tissue is associated with improvement in insulin resistance in a model of experimental diabetes. $J$ Endocrinol 2008, 198(1):51-60.

22. Smith GH, Medina D: Re-evaluation of mammary stem cell biology based on in vivo transplantation. Breast Cancer Res 2008, 10(1):203.

23. Daniel CW, Deome KB: Growth of Mouse Mammary Glands in Vivo after Monolayer Culture. Science 1965, 149(3684):634-636.

24. de Assis S WA, Cruz MI, Hilakivi-Clarke L: Changes in mammary gland morphology and breast cancer risk in rats. Journal of Visualized Experiments 2010(44).

25. Stanko JP, Fenton SE: Quantifying Branching Density in Rat Mammary Gland Whole-mounts Using the Sholl Analysis Method. J Vis Exp 2017(125).

26. Tucker DK, Foley JF, Bouknight SA, Fenton SE: Sectioning Mammary Gland Whole Mounts for Lesion Identification. J Vis Exp 2017(125).

27. de Assis S WA, Benitez C, Helferich W, Hilakivi-Clarke L: Protective effect of prepubertal genistein exposure on mammary tumorigenesis are dependent on BRCA1 expression. Cancer Prevention Research 2011, 4(9):1436-1448.

28. Elmore SA, Dixon D, Hailey JR, Harada T, Herbert RA, Maronpot RR, Nolte T, Rehg JE, Rittinghausen S, Rosol TJ et al: Recommendations from the INHAND Apoptosis/Necrosis Working Group. Toxicol Pathol 2016, 44(2):173-188.

29. Loudig O, Wang T, Ye K, Lin J, Wang Y, Ramnauth A, Liu C, Stark A, Chitale D, Greenlee R et al: Evaluation and Adaptation of a Laboratory-Based cDNA Library Preparation Protocol for Retrospective Sequencing of Archived MicroRNAs from up to 35-Year-Old Clinical FFPE Specimens. Int J Mol Sci 2017, 18(3).

30. Bolger AM LM, Usadel B: Trimmomatic: a flexible trimmer for illumina sequence data. Bioinformatics 2014, 30(15):2114-2120.

31. Clarke R, Ressom HW, Wang A, Xuan J, Liu MC, Gehan EA, Wang Y: The properties of highdimensional data spaces: implications for exploring gene and protein expression data. Nat Rev 
Cancer 2008, 8(1):37-49.

32. Ng SF LR, Barres R, Owens JA, Morris MJ: Chronic high-fat diet in fathers programs $\beta$-cell dysfunction in female rat offspring. Nature 2010, 467(7318):963-966.

33. Joshi PA, Waterhouse PD, Kasaian K, Fang H, Gulyaeva O, Sul HS, Boutros PC, Khokha R: PDGFRalpha(+) stromal adipocyte progenitors transition into epithelial cells during lobuloalveologenesis in the murine mammary gland. Nat Commun 2019, 10(1):1760.

34. Barcellos-Hoff MH, Ravani SA: Irradiated mammary gland stroma promotes the expression of tumorigenic potential by unirradiated epithelial cells. Cancer Res 2000, 60(5):1254-1260.

35. Arendt LM, Rudnick JA, Keller PJ, Kuperwasser C: Stroma in breast development and disease. Semin Cell Dev Biol 2010, 21(1):11-18.

36. Hovey RC, Aimo L: Diverse and active roles for adipocytes during mammary gland growth and function. J Mammary Gland Biol Neoplasia 2010, 15(3):279-290.

37. Akinyemiju T, Moore JX, Pisu M, Judd SE, Goodman M, Shikany JM, Howard VJ, Safford M, Gilchrist SC: A Prospective Study of Obesity, Metabolic Health, and Cancer Mortality. Obesity (Silver Spring) 2018, 26(1):193-201.

38. Bracci PM: Obesity and pancreatic cancer: overview of epidemiologic evidence and biologic mechanisms. Mol Carcinog 2012, 51(1):53-63.

39. Kerr J, Anderson C, Lippman SM: Physical activity, sedentary behaviour, diet, and cancer: an update and emerging new evidence. Lancet Oncol 2017, 18(8):e457-e471.

40. Li YR, Ro V, Tchou JC: Obesity, Metabolic Syndrome, and Breast Cancer: From Prevention to Intervention. Curr Surg Rep 2018, 6(3).

41. Chang HH, Moro A, Takakura K, Su HY, Mo A, Nakanishi M, Waldron RT, French SW, Dawson DW, Hines $\mathrm{OJ}$ et al: Incidence of pancreatic cancer is dramatically increased by a high fat, high calorie diet in KrasG12D mice. PLoS One 2017, 12(9):e0184455.

42. Cleary MP: Impact of obesity on development and progression of mammary tumors in preclinical models of breast cancer. J Mammary Gland Biol Neoplasia 2013, 18(3-4):333-343.

43. Doerstling SS, O'Flanagan CH, Hursting SD: Obesity and Cancer Metabolism: A Perspective on Interacting Tumor-Intrinsic and Extrinsic Factors. Front Oncol 2017, 7:216.

44. Shigematsu M, Honda S, Kirino Y: Transfer RNA as a source of small functional RNA. J Mol Biol Mol Imaging 2014, 1(2).

45. Schorn AJ, Martienssen R: Tie-Break: Host and Retrotransposons Play tRNA. Trends Cell Bio/ 2018, 28(10):793-806.

46. Cancer CGoHFiB: Familial breast cancer: collaborative reanalysis of individual data from $\mathbf{5 2}$ epidemiological studies including 58,209 women with breast cancer and 101,986 women without the disease. Lancet 2001, 358(9291):1389-1399.

47. Valberg M, Grotmol T, Tretli S, Veierod MB, Moger TA, Aalen OO: A hierarchical frailty model for familial testicular germ-cell tumors. Am J Epidemiol 2014, 179(4):499-506. 
48. D AAE: Models of genetic susceptibility to breast cancer. Oncogene 2006, 25(43):5898-5905.

49. Shifler Bowers K, Francis E, Kraschnewski JL: The dual burden of malnutrition in the United States and the role of non-profit organizations. Prev Med Rep 2018, 12:294-297.

Figures

a)

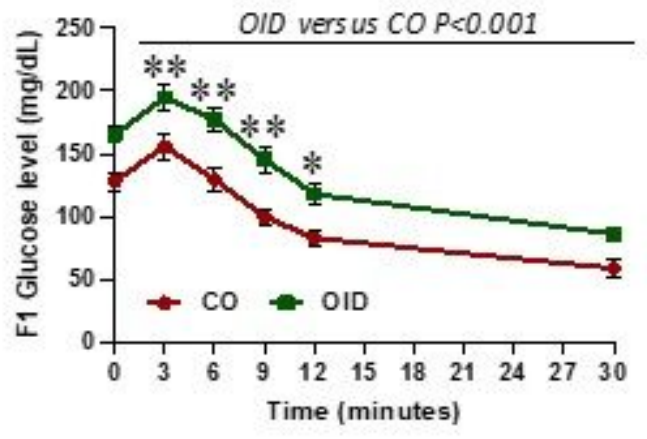

c)

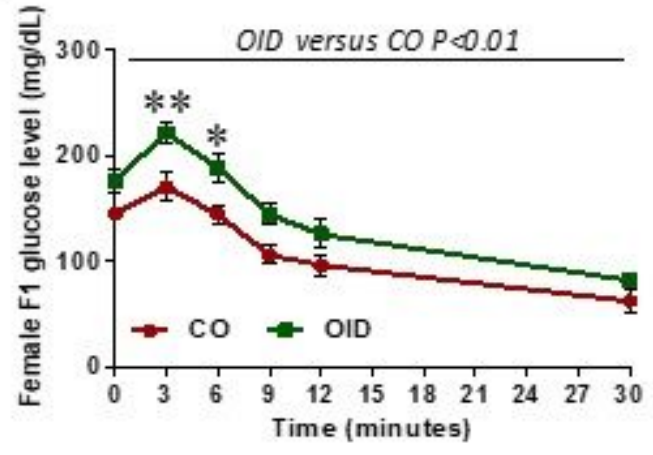

e)

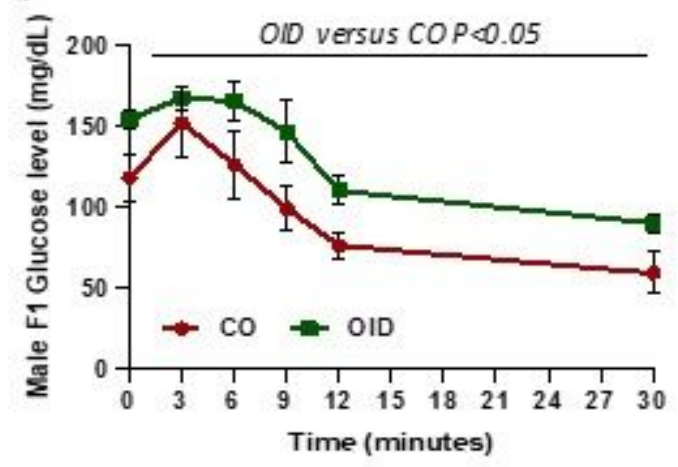

b)

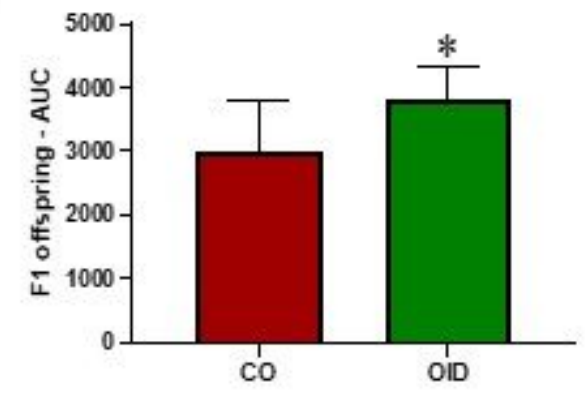

d)

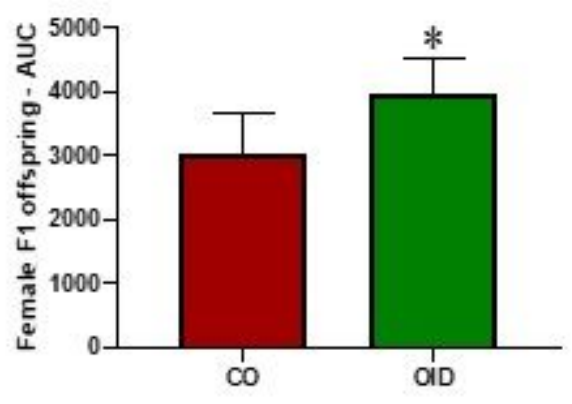

f)

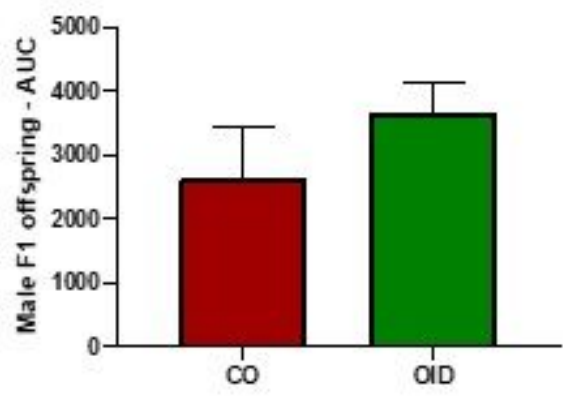

Figure 1

Figure 1 
Paternal OID causes metabolic disturbance in offspring. Insulin tolerance test (ITT) and area under curve (AUC) in all gender (a-b), female (c-d) and male (e-f) F1 offspring (n=7-8/gender/group) from CO and OIDfed fathers. The data are expressed as mean \pm SEM. Significant differences versus the control group were determined by two-way ANOVA followed by post-hoc analysis. ${ }^{*} \mathrm{P} \leq 0.05 ;{ }^{*} \mathrm{P} \leq 0.01$.

a)

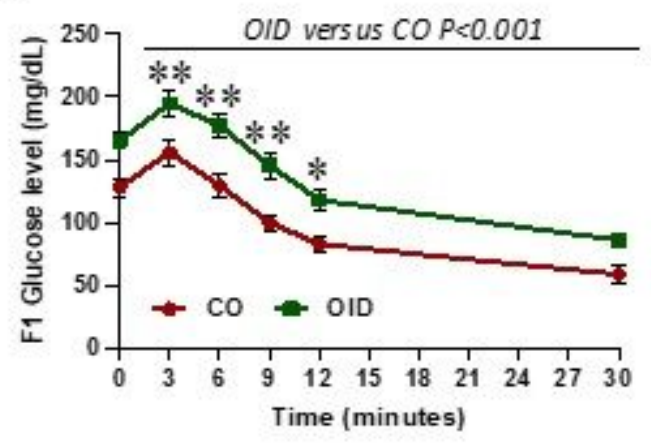

c)

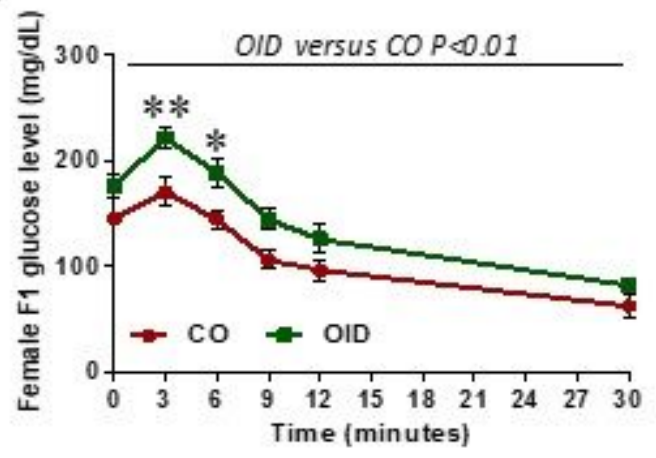

e)

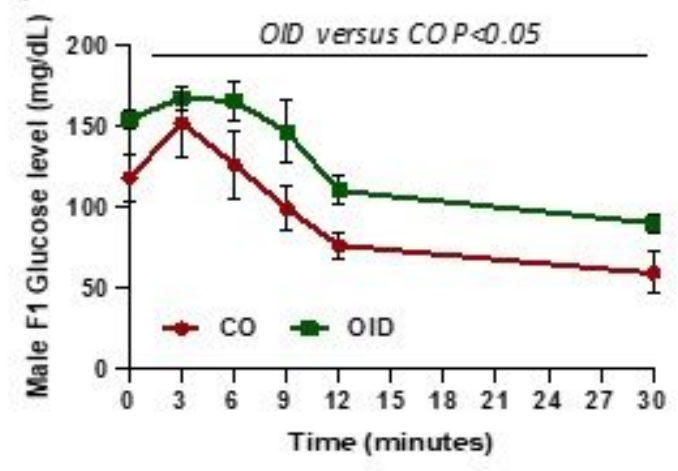

b)

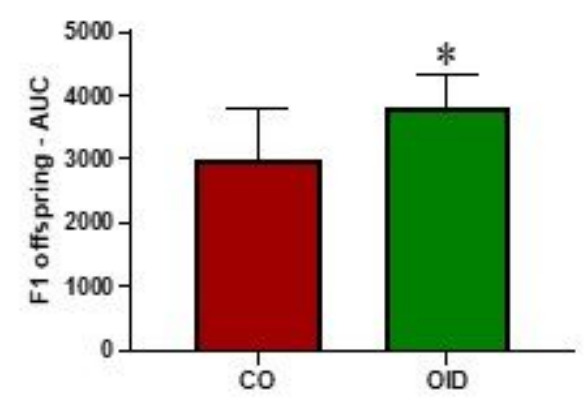

d)

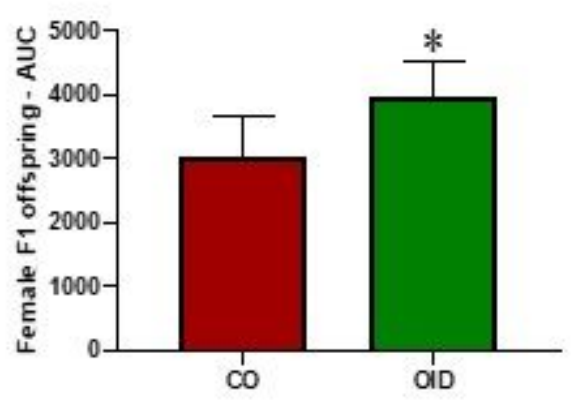

f)

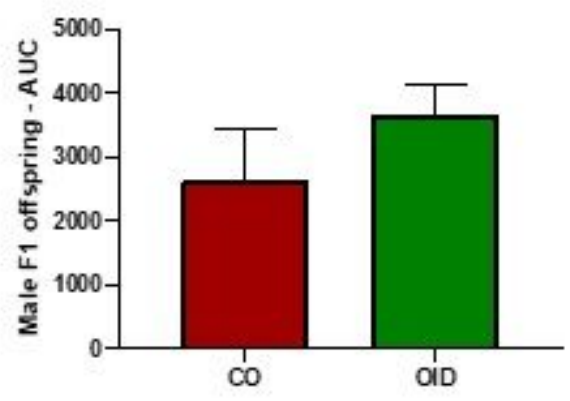

\section{Figure 1}

\section{Figure 1}

Paternal OID causes metabolic disturbance in offspring. Insulin tolerance test (ITT) and area under curve (AUC) in all gender (a-b), female (c-d) and male (e-f) F1 offspring ( $n=7-8 /$ gender/group) from CO and OID- 
fed fathers. The data are expressed as mean \pm SEM. Significant differences versus the control group were determined by two-way ANOVA followed by post-hoc analysis. ${ }^{*} \mathrm{P} \leq 0.05 ;{ }^{*} \mathrm{P} \leq 0.01$.

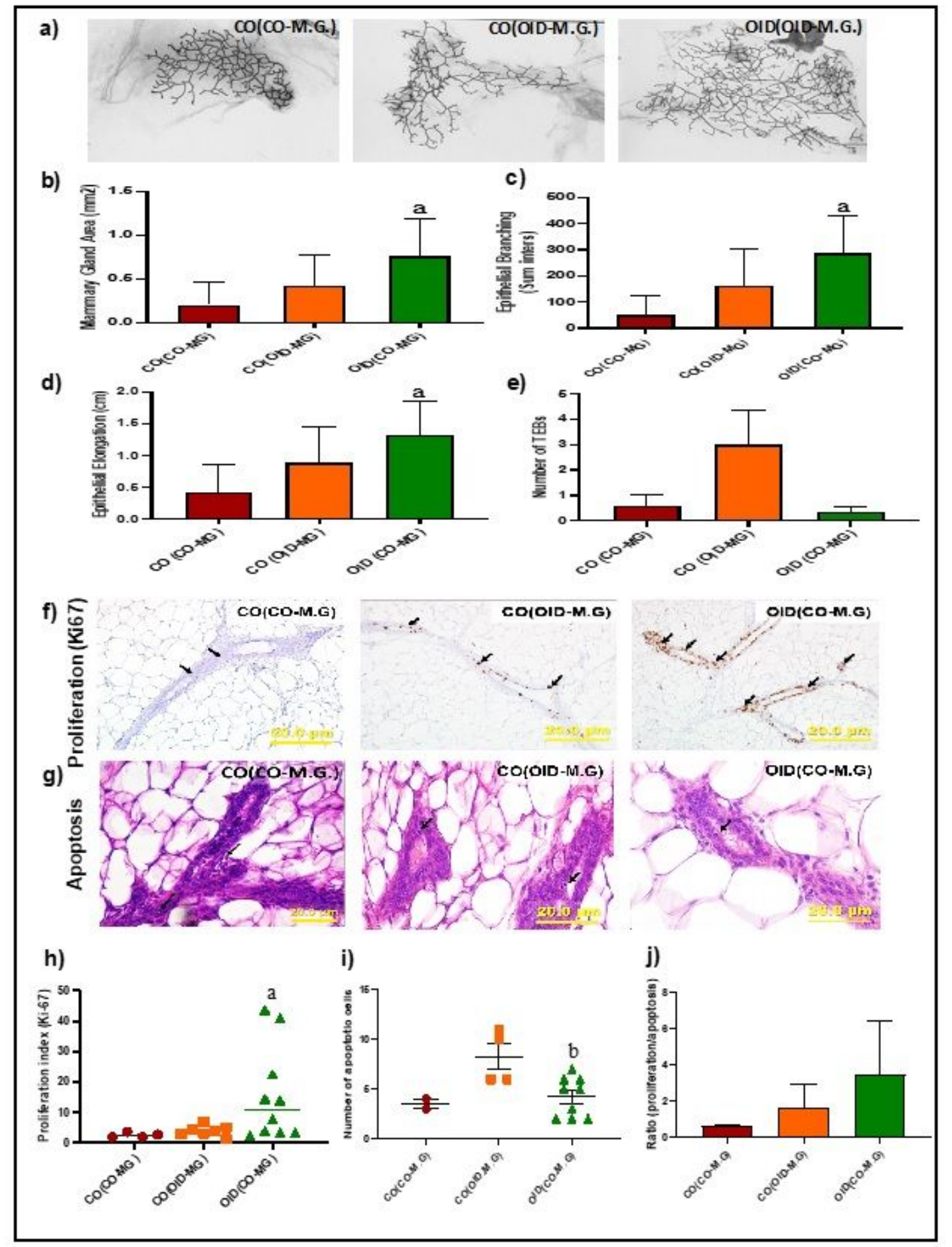

Figure 2

\section{Figure 2}

Development of transplanted mammary glands in CO or OID daughters (F1). Histological depiction of transplanted mammary gland in (a) [CO(CO-M.G)], [CO(OID-M.G)], and [OID(CO-M.G)] groups. Graphs below show values for mammary gland area (b), epithelial branching (c), epithelial elongation (d) and 
number of terminal end buds (TEB) (e), (b-e, $n=6-13)$; Photomicrograph of Ki-67 immunostaining (f) (20x, staining indicated by arrows) and apoptotic cells (g) (H\&E morphological assessment, 40x, cells indicated by arrows). Graphs below show proliferation index (h), number of apoptotic cells (i) and proliferation/apoptosis ratio (j), $(f-i, n=4-12)$. The data are expressed as mean \pm SEM. Significance differences between groups were determined by one-way ANOVA followed by post-hoc analysis (mammary gland area, branching density, epithelial elongation, number of TEBs, cell proliferation and apoptosis numbers). "a" indicates statistically significant difference ( $P \leq 0.05)$ between OID(CO-M.G) and CO(CO-M.G); " $b$ ” indicates statistically significant difference ( $\mathrm{P} \leq 0.05)$ between OID(CO-M.G) and CO(OIDM.G). 


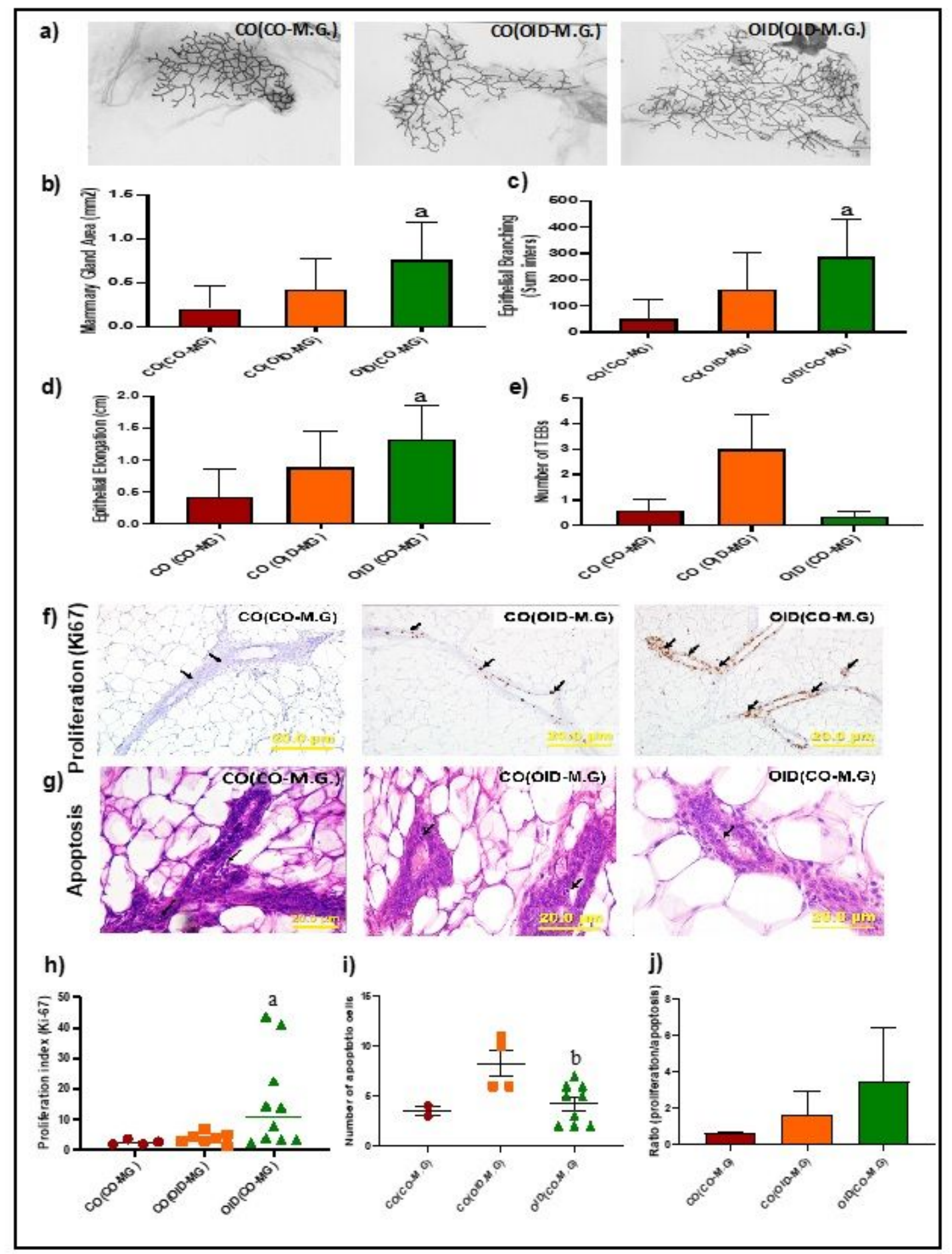

Figure 2

\section{Figure 2}

Development of transplanted mammary glands in CO or OID daughters (F1). Histological depiction of transplanted mammary gland in (a) [CO(CO-M.G)], [CO(OID-M.G)], and [OID(CO-M.G)] groups. Graphs below show values for mammary gland area (b), epithelial branching (c), epithelial elongation (d) and number of terminal end buds (TEB) (e), (b-e, $n=6-13)$; Photomicrograph of Ki-67 immunostaining (f) (20x, staining indicated by arrows) and apoptotic cells (g) (H\&E morphological assessment, 40x, cells indicated 
by arrows). Graphs below show proliferation index (h), number of apoptotic cells (i) and proliferation/apoptosis ratio $(j),(f-i, n=4-12)$. The data are expressed as mean $\pm S E M$. Significance differences between groups were determined by one-way ANOVA followed by post-hoc analysis (mammary gland area, branching density, epithelial elongation, number of TEBs, cell proliferation and apoptosis numbers). "a" indicates statistically significant difference ( $P \leq 0.05)$ between OID(CO-M.G) and CO(CO-M.G); "b" indicates statistically significant difference ( $\mathrm{P} \leq 0.05)$ between OID(CO-M.G) and CO(OIDM.G).

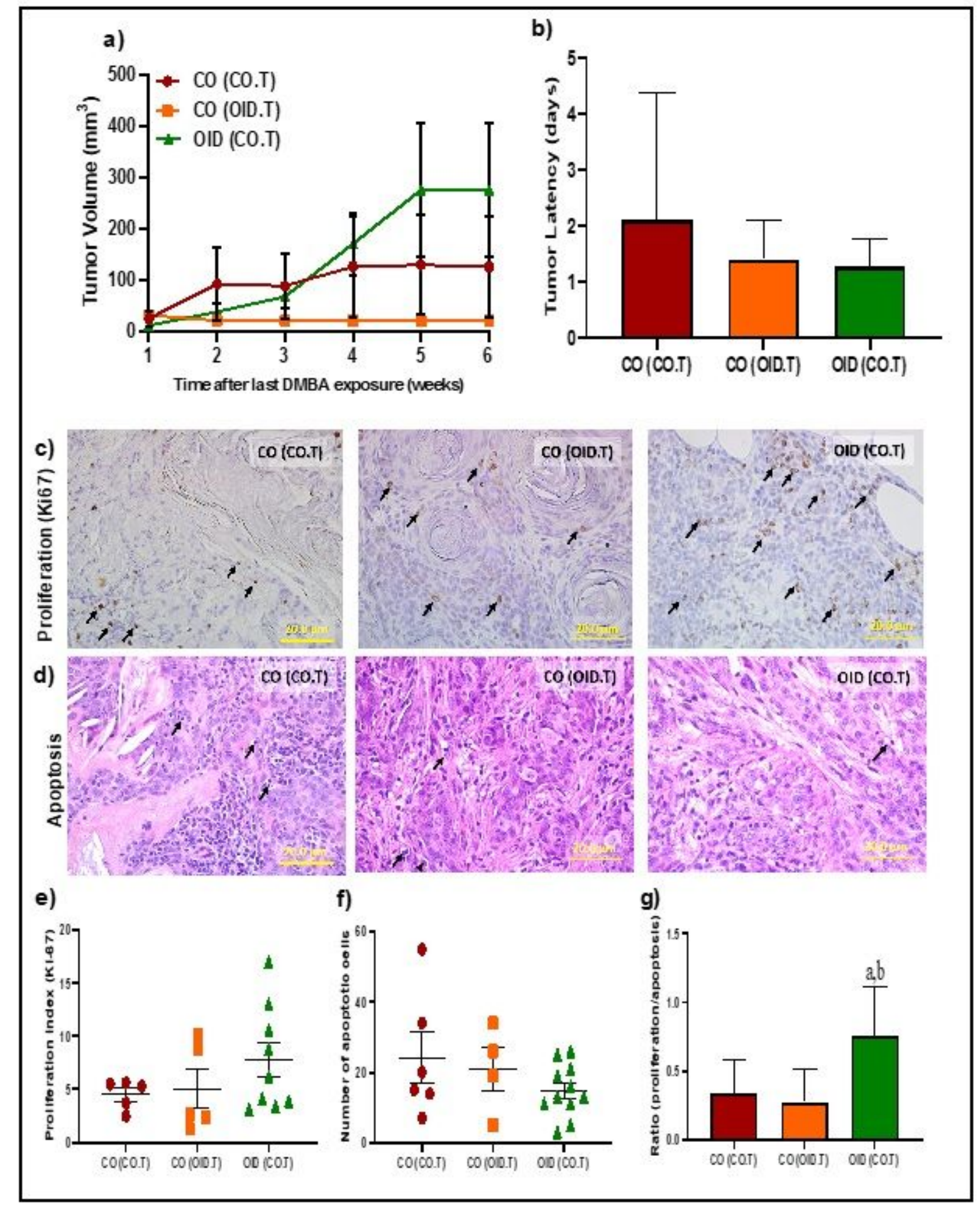

Figure 3 


\section{Figure 3}

Development of transplanted mammary tumors in CO or OID daughters (F1). Tumor volume (a) and latency (b) (a-b, n=10-18/group) in [CO(CO-M.G)], [CO(OID-M.G)], and [OID(CO-M.G)] groups after a sixweek monitoring period. Photomicrograph of Ki-67 immunostaining (c) (20x, staining indicated by arrows) and apoptotic cells (d) (H\&E morphological assessment, 20x, cells indicated by arrows). Graphs below show proliferation index (e), number of apoptotic cells $(\mathrm{f})$, and proliferation/apoptosis ratio (g), (c$g-n=3-11 /$ group). The data are expressed as mean \pm SEM. Significance differences between groups were analyzed by repeated measures ANOVA (mammary tumor volume) and one-way ANOVA (tumor latency, proliferation index and number of apoptotic cells) followed by post-hoc analysis. "a" indicates statistically significant difference $(\mathrm{P} \leq 0.05)$ between OID(CO.T) and CO(CO.T); "b" indicates statistically significant difference $(P \leq 0.05)$ between OID(CO.T) and CO(OID.T). 


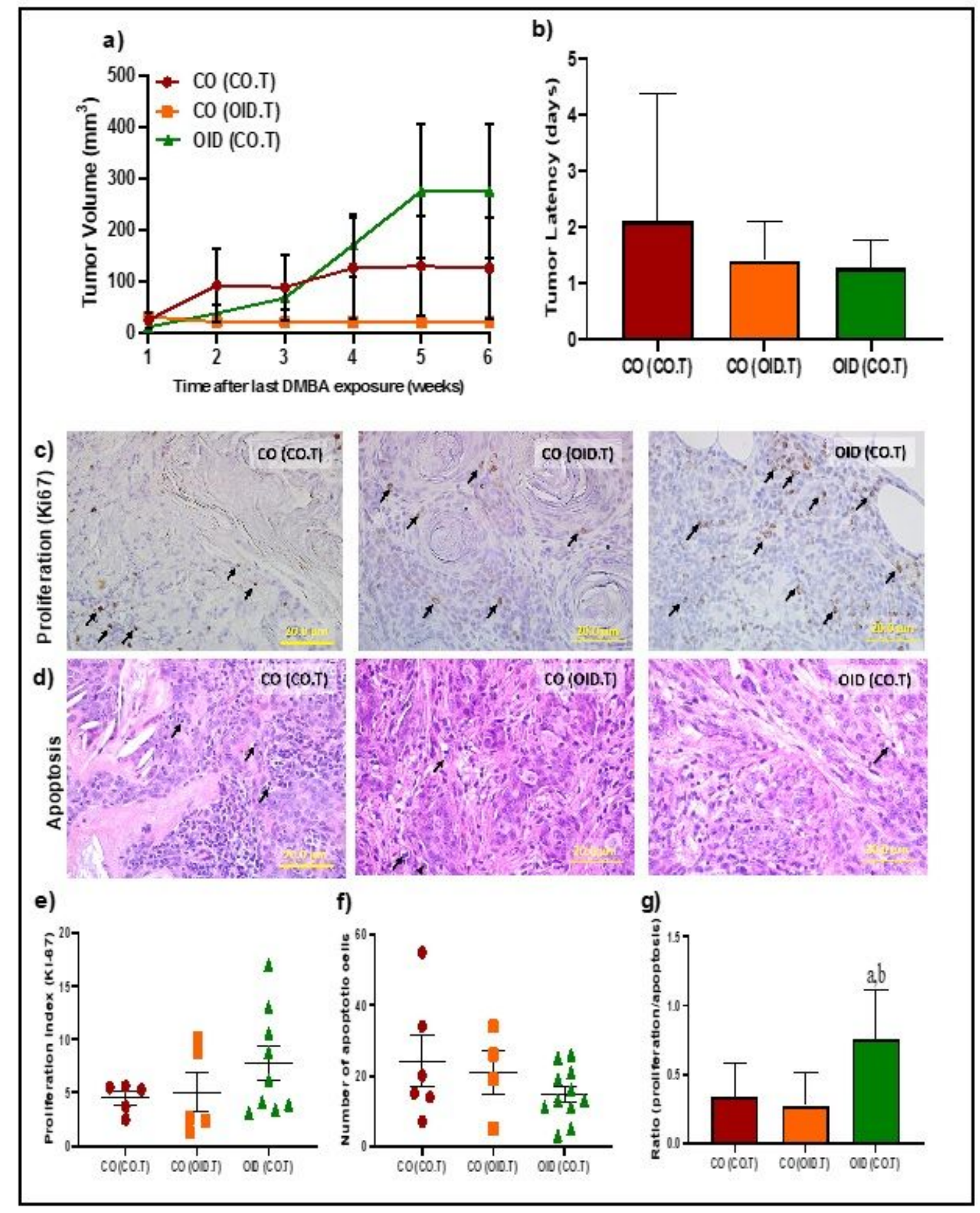

Figure 3

\section{Figure 3}

Development of transplanted mammary tumors in CO or OID daughters (F1). Tumor volume (a) and latency (b) (a-b, n=10-18/group) in [CO(CO-M.G)], [CO(OID-M.G)], and [OID(CO-M.G)] groups after a sixweek monitoring period. Photomicrograph of Ki-67 immunostaining (c) (20x, staining indicated by arrows) and apoptotic cells (d) (H\&E morphological assessment, 20x, cells indicated by arrows). Graphs below show proliferation index (e), number of apoptotic cells ( $\mathrm{f}$ ), and proliferation/apoptosis ratio (g), (c- 
$g-n=3-11 /$ group). The data are expressed as mean \pm SEM. Significance differences between groups were analyzed by repeated measures ANOVA (mammary tumor volume) and one-way ANOVA (tumor latency, proliferation index and number of apoptotic cells) followed by post-hoc analysis. "a" indicates statistically significant difference $(P \leq 0.05)$ between OID(CO.T) and CO(CO.T); " $b$ " indicates statistically significant difference $(\mathrm{P} \leq 0.05)$ between OID(CO.T) and CO(OID.T).

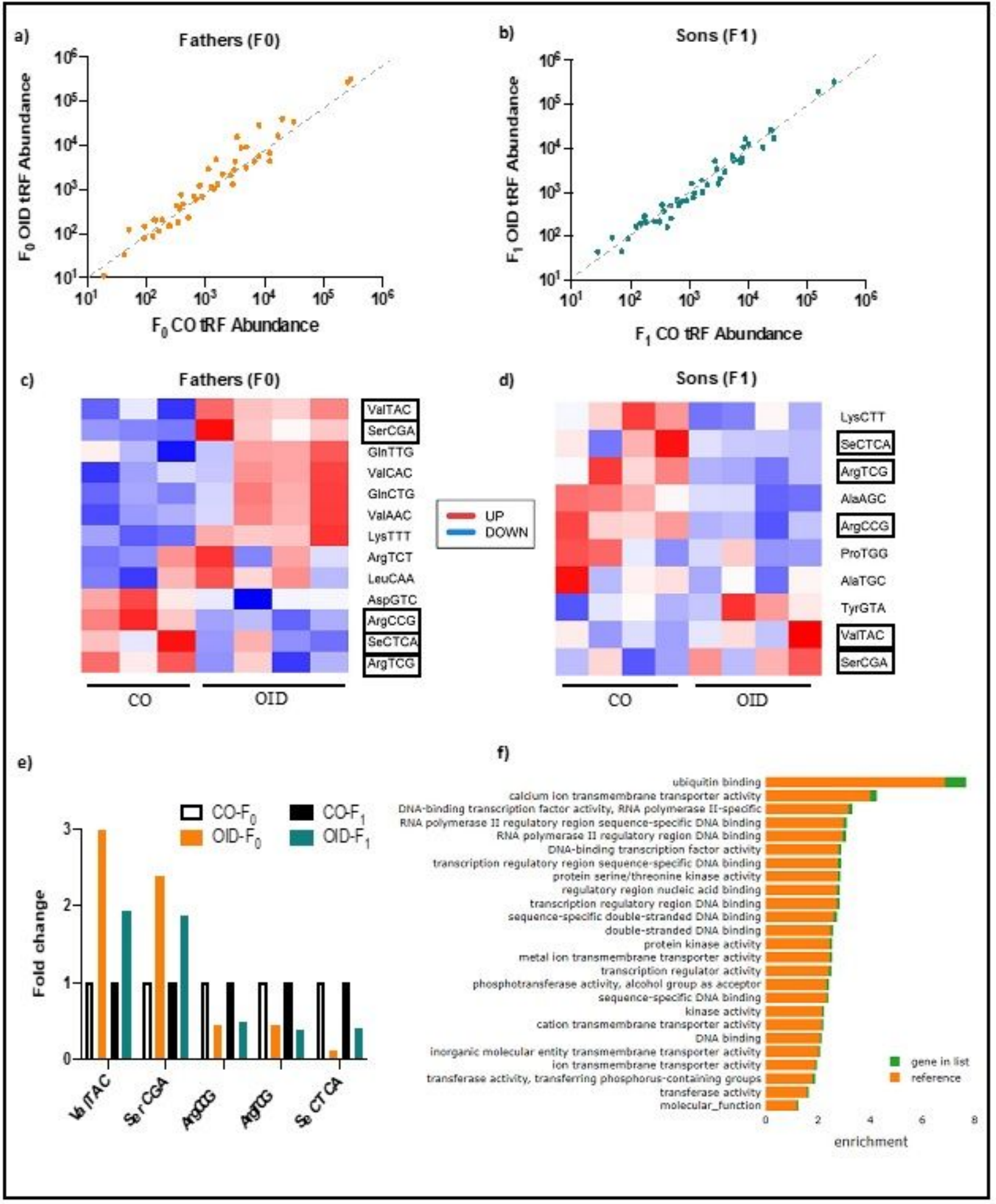

Figure 4

Figure 4 
Paternal OID reprograms the sperm small non-coding RNA load in fathers (F0) and sons (F1). (a-b) Scatterplot of sperm tRNA fragments (tRF) from OID (y-axis) fathers ( $F 0, a)$ and OID sons $(F 1, b)$ versus their respective controls ( $\mathrm{CO}, \mathrm{x}$-axis) ( $\mathrm{n}=3-4 /$ group) assessed by RNA-seq. (c-d) Heat-map showing differentially expressed tRNA fragments (tRFs) in sperm from OID fathers (c) and sons (d) compared to $\mathrm{CO}$, highlighting overlapping tRFs in F0 and F1 (boxes). (e) Levels (fold change) of the 5 tRFs with overlapping differential expression in both OID fathers(F0) and sons (F1) compared to $\mathrm{CO}$. (f) Gene ontology molecular functions significantly enriched in the targets of ValTAC, SerCGA, ArgCCG, ArgTCG and SeCTCA. 


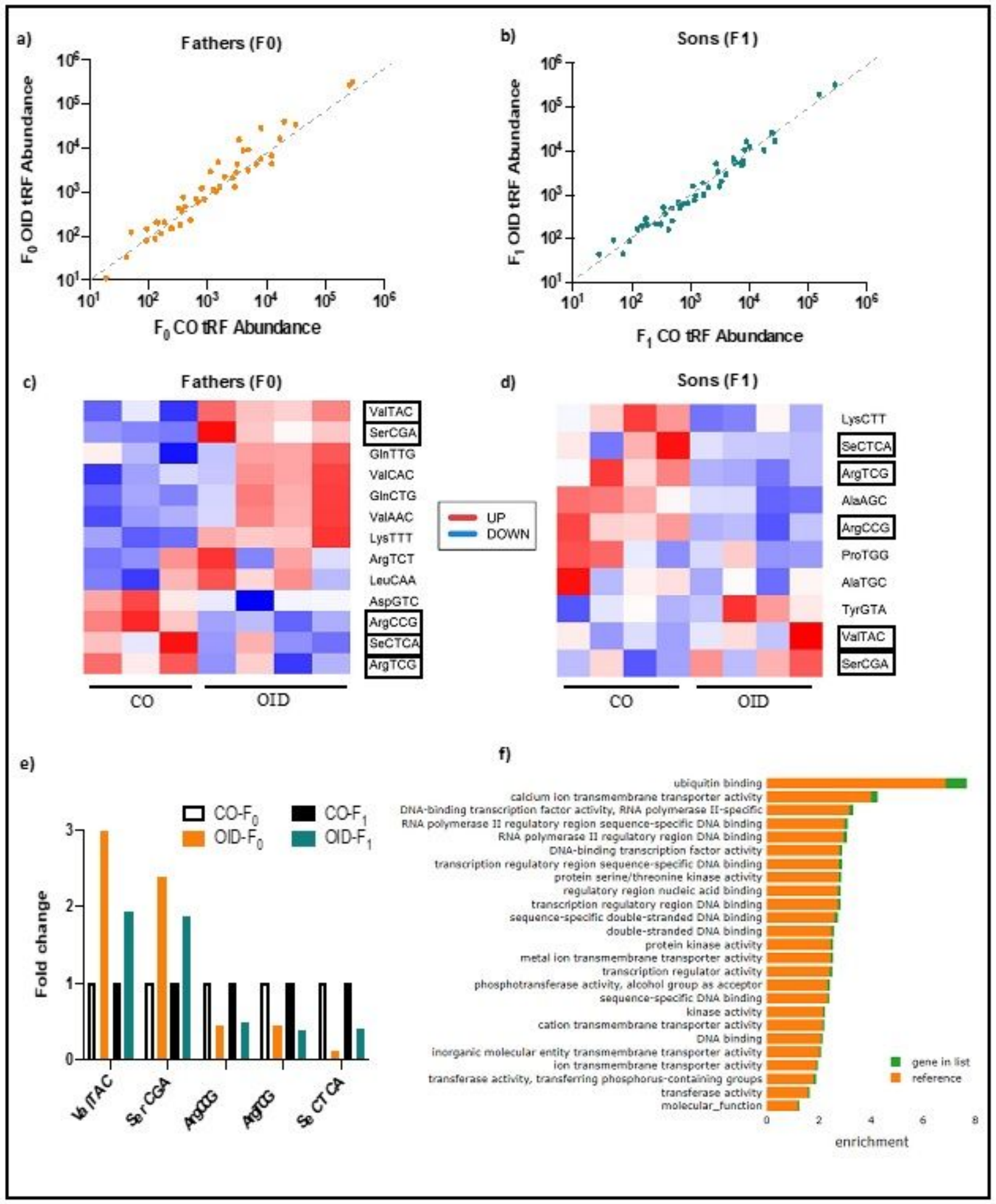

Figure 4

\section{Figure 4}

Paternal OID reprograms the sperm small non-coding RNA load in fathers (F0) and sons (F1). (a-b) Scatterplot of sperm tRNA fragments (tRF) from OID (y-axis) fathers (F0, a) and OID sons ( $F 1, b)$ versus their respective controls ( $\mathrm{CO}, \mathrm{x}$-axis) ( $\mathrm{n}=3-4 /$ group) assessed by RNA-seq. (c-d) Heat-map showing differentially expressed tRNA fragments (tRFs) in sperm from OID fathers (c) and sons (d) compared to $\mathrm{CO}$, highlighting overlapping tRFs in F0 and F1 (boxes). (e) Levels (fold change) of the 5 tRFs with 
overlapping differential expression in both OID fathers(F0) and sons (F1) compared to CO. (f) Gene ontology molecular functions significantly enriched in the targets of ValTAC, SerCGA, ArgCCG, ArgTCG and SeCTCA.

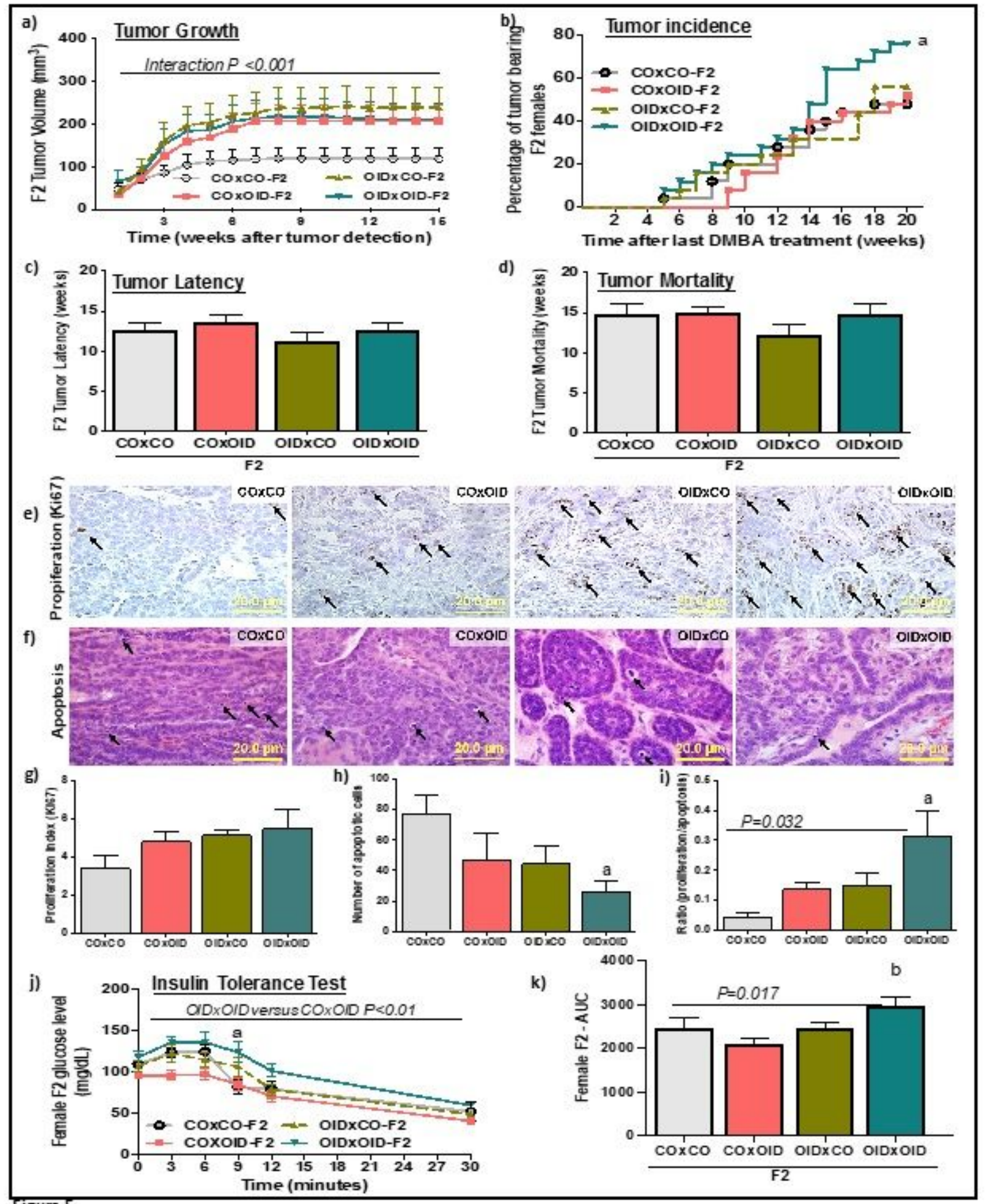

Figure 5

Figure 5

Paternal OID programs breast cancer development and metabolic dysfunction in granddaughters (F2). (a-

d) Carcinogen-induced mammary tumorigenesis in CO and OID female F2 offspring. Mammary tumor 
growth (volume, mm3; a), tumor incidence (b), tumor latency (c) and tumor mortality (d) ( $n=25 /$ group). Photomicrograph of Ki-67 immunostaining (e) (20x, staining indicated by arrows) and apoptotic cells ( $f$ ) (H\&E morphological assessment, 20x, cells indicated by arrows). Graphs below show proliferation index (g), number of apoptotic cells (h), and proliferation/apoptosis ratio (i), (e-i-n=6/group). Insulin tolerance test (ITT) (j) and ( $k$ ) area under curve (AUC) in CO and OID female F2 offspring ( $n=8 /$ group). Tumor incidence is shown as percentage of animals with tumors. All other data are mean \pm SEM. Significant difference were determined by Kaplan-Meier analysis followed by log-rank test (tumor incidence), repeated measures ANOVA (mammary tumor volume), one-way ANOVA (tumor latency, mortality, proliferation, apoptosis and area under curve), or two-way ANOVA (ITT) followed by post-hoc analysis. "a" indicates statistically significant difference $(\mathrm{P} \leq 0.05)$ between OIDxOID and COxCO; "b" indicates statistically significant difference $(P \leq 0.01)$ between OIDxOID and COxOID. 


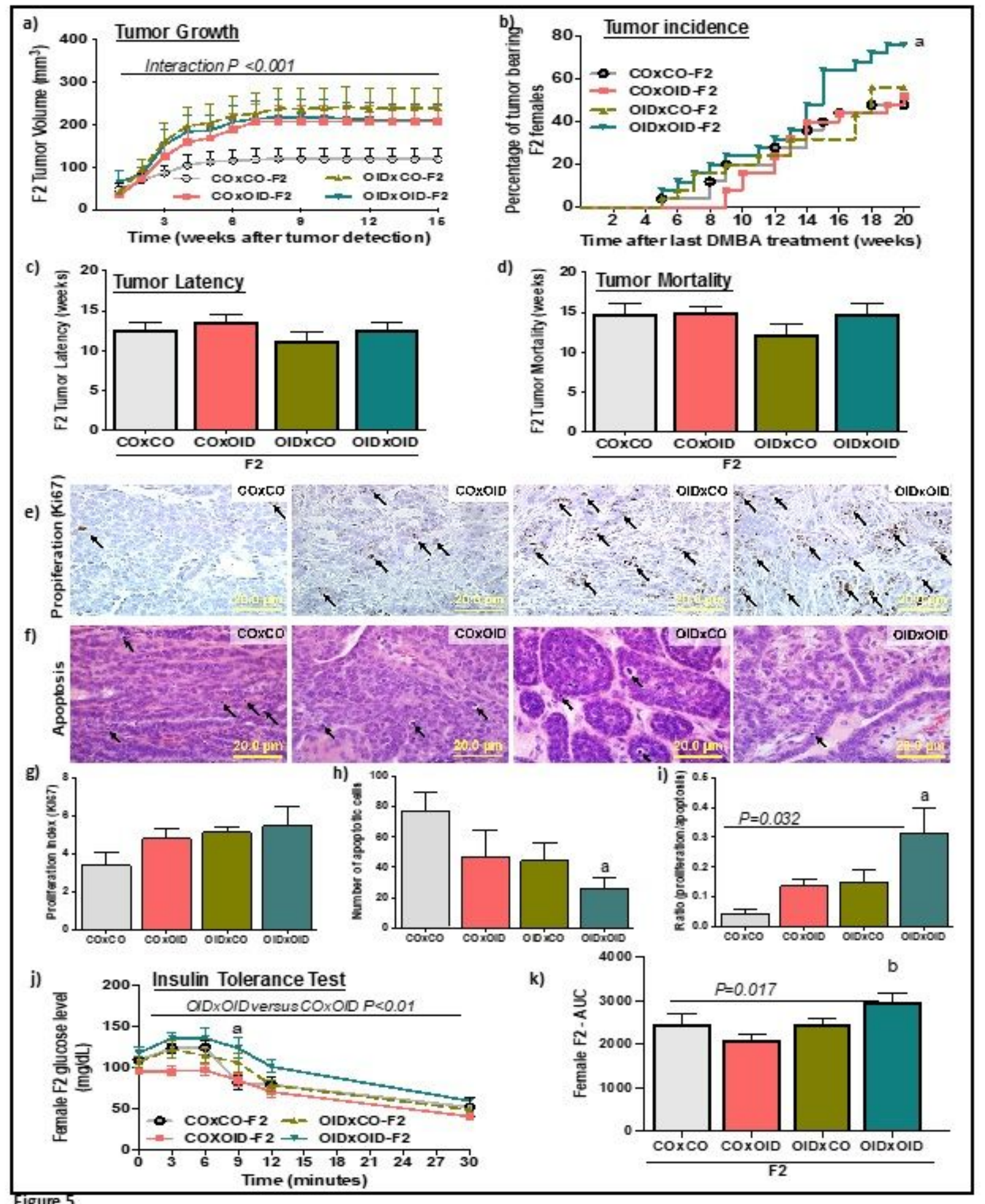

Figure 5

\section{Figure 5}

Paternal OID programs breast cancer development and metabolic dysfunction in granddaughters (F2). (ad) Carcinogen-induced mammary tumorigenesis in $\mathrm{CO}$ and OID female F2 offspring. Mammary tumor growth (volume, mm3; a), tumor incidence (b), tumor latency (c) and tumor mortality (d) ( $n=25 /$ group). Photomicrograph of Ki-67 immunostaining (e) (20x, staining indicated by arrows) and apoptotic cells (f) (H\&E morphological assessment, 20x, cells indicated by arrows). Graphs below show proliferation index 
(g), number of apoptotic cells (h), and proliferation/apoptosis ratio (i), (e-i-n=6/group). Insulin tolerance test (ITT) (j) and ( $k$ ) area under curve (AUC) in CO and OID female F2 offspring ( $n=8 /$ group). Tumor incidence is shown as percentage of animals with tumors. All other data are mean \pm SEM. Significant difference were determined by Kaplan-Meier analysis followed by log-rank test (tumor incidence), repeated measures ANOVA (mammary tumor volume), one-way ANOVA (tumor latency, mortality, proliferation, apoptosis and area under curve), or two-way ANOVA (ITT) followed by post-hoc analysis. "a" indicates statistically significant difference $(P \leq 0.05)$ between OIDxOID and COxCO; "b" indicates statistically significant difference $(P \leq 0.01)$ between OIDxOID and COxOID.

\section{Supplementary Files}

This is a list of supplementary files associated with this preprint. Click to download.

- SUPPLEMENTARYINFORMATION.docX

- SUPPLEMENTARYINFORMATION.docX 